\title{
The comparison of extraction methods of ganjiang decoction based on fingerprint, quantitative analysis and pharmacodynamics
}

Yanyan Wei ${ }^{1 \dagger}$, Ning Jiang ${ }^{1 \dagger}$, Tuo Liu', Chang Liu', Wen Xiao ${ }^{1}$, Likeng Liang ${ }^{2}$, Tongming $\mathrm{Li}^{1 *}$ and Yang Yu ${ }^{1 *}$

\begin{abstract}
Background: Ulcerative colitis (UC) is a chronic nonspecific inflammatory disease of the colon and rectum with unknown etiology, and its symptoms include bloody diarrhea, abdominal pain, and hematochezia. Traditional Chinese medicine compound has a good therapeutic, multi-target effect on UC. Ganjiang decoction (GD), which is a traditional classic prescription in China, contains Zingiberis Rhizoma, Angelicae Sinensis Radix, Coptidis Rhizoma, Phellodendri Chinensis Cortex, Sanguisorbae Radix, Granati Pericarpium, and Asini Corii Colla and could be used to treat symptoms of UC. This study aimed to conduct a preliminary study before GD colon-targeted preparation, to explore the relationship between extraction method and efficacy of GD.

Methods: High-performance liquid chromatography (HPLC) was used for the fingerprinting of five preparation methods of GD. HPLC and gas chromatography were used to quantitatively analyze the important chemical components of GD and compare their differences. Mice with $U C$ induced by dextran sulphate sodium salt received the extracts from the five preparation methods of GD via gavage. Disease activity index (DAI) score, colonic length, relative weight of spleen, pathological analysis results, inflammatory factors, therapeutic effect of the five preparation methods of GD, and their relationship with extraction process were compared.
\end{abstract}

Results: Cluster analysis revealed that the content of the components extracted by traditional extraction methods was significantly different from the other four methods. The third and fifth preparation methods extracted Coptidis Rhizoma and Phellodendri Chinensis Cortex with 50\% ethanol to obtain more alkaloids. In the fourth and fifth methods, more volatile oils were detected by adding Zingiberis Rhizoma and Angelicae Sinensis Radix fine powder. According to DAl score, colonic length, relative weight of spleen, pathological analysis results, and inflammatory factors, the third method showed a good therapeutic effect, while the fifth method had the best therapeutic effect.

Conclusions: The results showed that the difference of the five extracts of GD in the efficacy of DSS-induced UC in mice was closely related to the extraction method. Our study improved the extraction process of GD and provided a foundation for the process of enteric-soluble preparations and a new idea for traditional Chinese medicine compound preparation.

Keywords: Ganjiang decoction, Fingerprint, Quantitative analysis, Pharmacodynamics

\footnotetext{
*Correspondence: Itm@gzucm.edu.cn; yuyang@gzucm.edu.cn

${ }^{\dagger}$ Yanyan Wei and Ning Jiang contributed equally to this work

${ }^{1}$ School of Pharmaceutical Sciences, Guangzhou University of Chinese

Medicine, Guangzhou, Guangdong 510006, China

Full list of author information is available at the end of the article
}

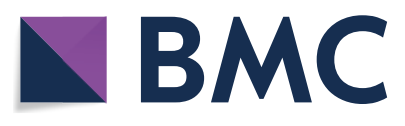

(c) The Author(s) 2020. This article is licensed under a Creative Commons Attribution 4.0 International License, which permits use, sharing, adaptation, distribution and reproduction in any medium or format, as long as you give appropriate credit to the original author(s) and the source, provide a link to the Creative Commons licence, and indicate if changes were made. The images or other third party material in this article are included in the article's Creative Commons licence, unless indicated otherwise in a credit line to the material. If material is not included in the article's Creative Commons licence and your intended use is not permitted by statutory regulation or exceeds the permitted use, you will need to obtain permission directly from the copyright holder. To view a copy of this licence, visit http://creativeco mmons.org/licenses/by/4.0/. The Creative Commons Public Domain Dedication waiver (http://creativecommons.org/publicdomain/ zero/1.0/) applies to the data made available in this article, unless otherwise stated in a credit line to the data. 


\section{Background}

Ganjiang decoction (GD), which is a Chinese herbal formula, was first recorded in the Tang Dynasty 'ThousandGolden-Prescriptions (Beiji Qianjin Yao Fang)' volume 14, prescription of Zingiberis Rhizoma, Angelicae Sinensis Radix, Coptidis Rhizoma, Phellodendri Chinensis Cortex, Sanguisorbae Radix, Granati Pericarpium, and Asini Corii Colla. Clinically, it was mainly used for the treatment of small intestine distension and pain, hematochezia or loose stools, and intestinal peristalsis, which are clinical manifestations similar to those of ulcerative colitis (UC). The combination of traditional Chinese medicinal materials played different roles in the prescription of GD. Zingiberis Rhizoma and Angelicae Sinensis Radix were used as analgesic and anti-inflammatory agents [1]. Coptidis Rhizoma and Phellodendri Chinensis Cortex showed an anti-inflammatory and bacteriostatic effect [2-6]. Sanguisorbae Radix and Granati Pericarpium had anti-inflammatory and anti-astringent effects $[7,8]$.

$\mathrm{UC}$, which is a type of inflammatory bowel disease, is an inflammation of the colon with recurrent attacks and lesions mainly affecting the mucous membranes of the colon and rectum. Currently, the incidence of UC has increased worldwide, with a prevalence of 24.3, 19.2, and 6.3 in 100,000 people in Europe, North America, and Asia, respectively $[9,10]$. It has been listed as one of the modern intractable diseases by the World Health Organization.

Although the exact etiology and pathogenesis of UC remain unclear, UC is considered an autoimmune disease, which is affected by multiple factors and closely related to genetic, environmental, immune, and inflammatory mediators; intestinal microecological disorder; intestinal mucosal barrier injury; cytokine imbalance; and other factors [11]. As the mechanism of UC needs to be clarified, appropriate drug therapy remains to be established. Currently, chemical drugs used to treat UC mainly include aminosalicylic acid, glucocorticoids, and immunosuppressants, whose effects are not satisfactory. Moreover, these agents are associated with relapse and more long-term side effects $[12,13]$.

Traditional Chinese medicine compound contains a variety of ingredients and thus has multi-targeted effect and certain advantages [14-16]. Ancient Chinese medicine used the human body to evaluate the effect of drug use, and oral decoction was primarily administered. However, understanding of the etiology and pathology was limited, and an oral decoction also had its limitations. Moreover, traditional decoction was often extracted by water, and the active ingredients were not dissolved completely, which limited the curative effect. UC occurs in the sigmoid colon and colon; thus, the effect of traditional decoction could not reach the affected area. Hence, traditional Chinese medicine development has always aimed to improve pharmacological, chemical, and targeted drug delivery to increase patients' treatment compliance.

According to the clinical needs, GD is intended to be developed as a colon-targeted agent. This study aimed to conduct a preliminary study before GD colon-targeted preparation, to explore the relationship between extraction method and efficacy of GD. High-performance liquid chromatography (HPLC) was used for the fingerprinting of five preparation methods of GD, and HPLC and gas chromatography (GC) were used to compare the differences of the chemical components, thereby providing a foundation for the process of enteric-soluble preparations. Moreover, the therapeutic effects of the five preparation methods of GD on experimental colitis mice were compared. This study also provided a new idea for traditional Chinese medicine compound preparation.

\section{Materials and methods Chemicals and reagents}

Zingiberis Rhizoma, Angelicae Sinensis Radix, Coptidis Rhizoma, Phellodendri Chinensis Cortex, Sanguisorbae Radix, and Granati Pericarpium were identified by Dr. Peng Guangtian of Guangzhou University of Chinese Medicine (Fig. 1). Zingiberis Rhizoma, Angelicae Sinensis Radix, and Granati Pericarpium were purchased from Anhui Jishun traditional Chinese Medicine Co., Ltd. (Bozhou, China); Coptidis Rhizoma and Sanguisorbae Radix were purchased from Anhui Jucao Traditional Chinese Medicine Co., Ltd. (Bozhou, China); Phellodendri Chinensis Cortex was purchased from Shanxi HongSen Traditional Chinese Medicine Co., Ltd. (Xian, China); Asini Corii Colla was purchased from Shandong Jishui Ejiao Co., Ltd. (Heze, China). Zingiberone, ferulic acid, palmatine, jatrorrhizine, berberine hydrochloride, gallic acid, ellagic acid, zingerone and ligustilide had a purity of $98 \%$, as determined by HPLC or GC, and were provided by Chengdu Weikeqi Biotechnology Co. Ltd. (Chengdu, China). Methanol and acetonitrile were purchased from Merck \& Co. Inc. (Darmstadt, Germany). Phosphoric acid was purchased from Aladdin Reagent Co., Ltd. (Shanghai, China). Dextran sulphate sodium salt (DSS) was provided by Yisheng Biotechnology Co., Ltd. (Shanghai, China). Salicylazosulfapyridine (SASP) enteric-coated tablets were provided by Fuda Pharmaceutical Co., Ltd. (Shanghai, China). All other chemicals were of reagent grade. 


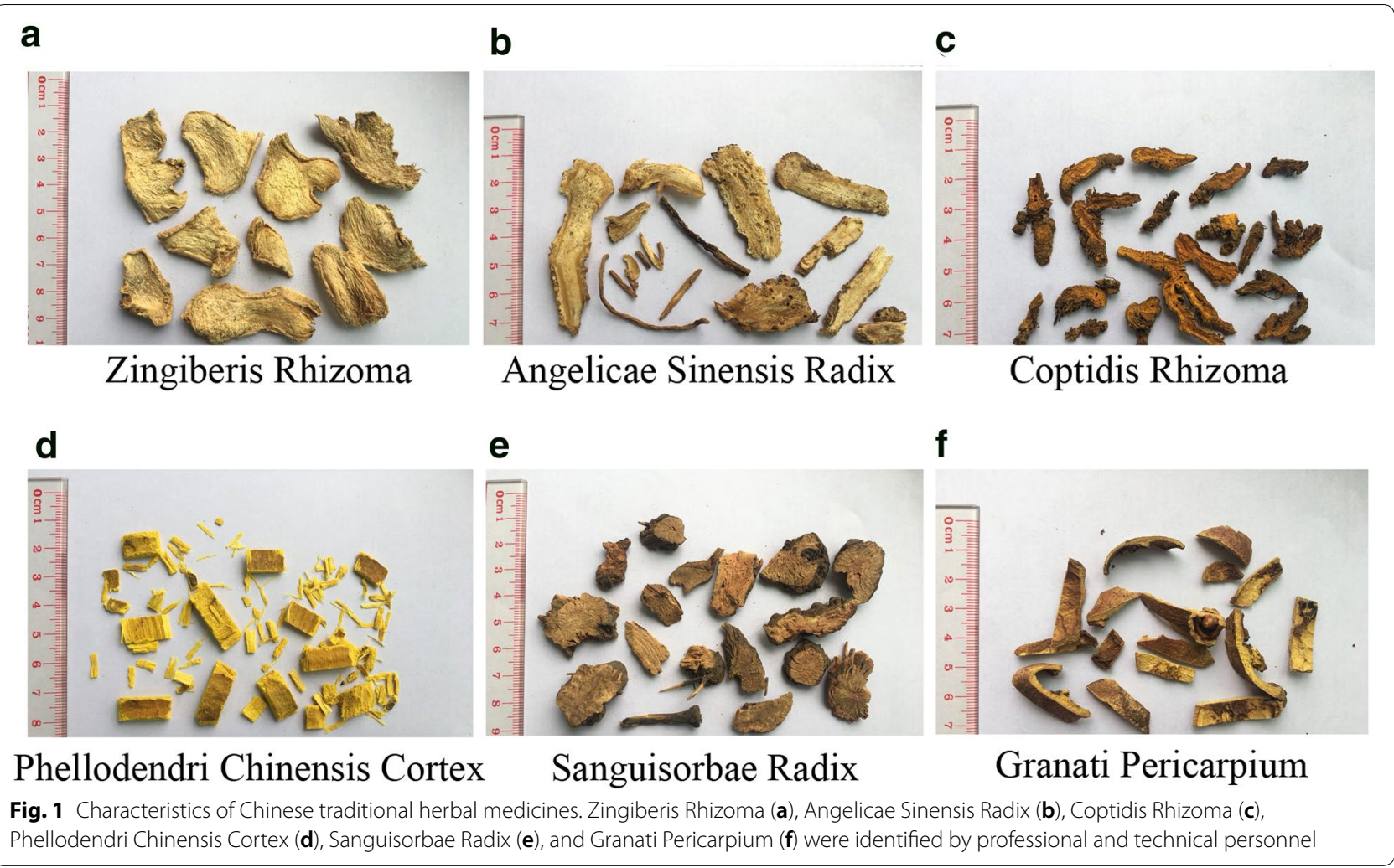

\section{Five preparation methods of ganjiang decoction}

GD can be divided into four combinations according to the property, flavor, and pharmacologic action of traditional Chinese medicine. Zingiberis Rhizoma and Angelicae Sinensis Radix are the combination 1, for the warm medicine group. Coptidis Rhizoma and Phellodendri Chinensis Cortex are combination 2, which is the cold medicine group. Combination 3 consist of Sanguisorbae Radix and Granati Pericarpium, which is the astringent group. Combination 4, there is Asini Corii Colla, which is the hemostatic group.

According to the property and flavor of these traditional Chinese medicine and the character of the ingredients contained, GD was prepared by five methods. Method 1 was to extract the whole compound prescription with distilled water. Coptidis Rhizoma and Phellodendri Chinensis Cortex, Sanguisorbae Radix and Granati Pericarpium were extracted respectively in methods $2-5$ to prevent the reaction between components. Methods 2-5 were also investigated the effects of different solvents on the extraction efficiency of alkaloids. Some fine powder of Zingiberis Rhizoma and Angelicae Sinensis Radix was added in method 4 and method 5 to increase the volatile oil. The specific preparation method of GD was shown in Table 1.

\section{Fingerprint analysis of ganjiang decoction prepared by the five methods}

The final sample obtained by the five extraction methods was the mixture of dried extract and dried medicinal powder. Methanol was added to the samples prepared by the five methods and ultrasonic for $30 \mathrm{~min}$. The supernatant solution was obtained by a $0.22 \mu \mathrm{m}$ microporous filtration membrane.

The fingerprint and quantitative analyses of GD by HPLC were performed by an LC-20AT HPLC System and LabSolutions chromatographic work station (SHIMADZU Cooperation, Japan); Waters- $\mathrm{C}_{18}$ column $(250 \mathrm{~mm} \times 4.6 \mathrm{~mm} \times 5 \mu \mathrm{m})$ (Waters Cooperation, US) was used. The mobile phases of the fingerprint analysis were composed of methanol (A) $-0.1 \%$ phosphoric acid water (B), with gradient elution $(0-5 \mathrm{~min}$, A $10-15 \%$; $5-25$ min, A $15-39 \%$; $25-38$ min, A $39-45 \%$; $38-40 \mathrm{~min}, \mathrm{~A} 45-48 \%$; $40-50 \mathrm{~min}$, A $48-48 \%$; $50-55 \mathrm{~min}, \mathrm{~A} 48-60 \%$ ) at a flow rate of $0.8 \mathrm{~mL} / \mathrm{min}$. The column temperature was $30{ }^{\circ} \mathrm{C}$. The detection UV wavelength was $254 \mathrm{~nm}$, and the injection volume was $10 \mu \mathrm{L}$.

The data of 10 batches of samples for five extraction method were imported into the similarity evaluation system software of traditional Chinese medicine chromatographic fingerprint (Version 2012.130723). S1 was 
Table 1 Different preparation method of ganjiang decoction

\begin{tabular}{|c|c|c|c|}
\hline \multirow[t]{2}{*}{ Method } & \multicolumn{3}{|l|}{ Extraction } \\
\hline & $\begin{array}{l}\text { Reflux extracted with distilled water } \\
\text { for } 30 \text { min twice, decompressed } \\
\text { subsequently, concentrated to a thickening } \\
\text { paste, dried at } 60^{\circ} \mathrm{C} \text {. }\end{array}$ & $\begin{array}{l}\text { Reflux extracted with } 50 \% \text { alcohol } \\
\text { for } 60 \text { min, decompressed subsequently, } \\
\text { concentrated to a thickening paste, dried } \\
\text { at } 60^{\circ} \mathrm{C} \text {. }\end{array}$ & Fine powder \\
\hline 1 & Extract combination (1)(2)(3) together & & Combination (4) \\
\hline 2 & $\begin{array}{l}\text { Extract combination (1)(3) together, } \\
\text { Extract combination (2) separately }\end{array}$ & & Combination (4) \\
\hline 3 & Extract combination (1)(3) together & Combination (2) & Combination (4) \\
\hline 4 & $\begin{array}{l}\text { Extract combination (1)(3) together, } \\
\text { Extract combination (2) separately }\end{array}$ & & A little Combination (1), Combination (4) \\
\hline 5 & Extract combination (1)(3) together & Combination (2) & A little Combination (1), Combination (4) \\
\hline
\end{tabular}

Combination (1): Zingiberis Rhizoma and Angelicae Sinensis Radix; Combination (2): Coptidis Rhizoma and Phellodendri Chinensis Cortex; Combination (3): Sanguisorbae Radix and Granati Pericarpium. Combination (4): Asini Corii Colla. The total quantity of Combination (1) in method 4 and method 5 was the same as that in other methods. The amount of fine powder added was one-sixth of the amount extracted by solvent

set as the reference map, the whole spectrum peak was automatically matched, the control map was generated, and the similarity was calculated.

The peak area of each component was standardized, and hierarchical clustering analysis was performed on the standardized data. In the hierarchical cluster analysis, Euclidean method was adopted. Distance was measured for similarity, and the clustering method was complete linkage. The clustering results were shown in heat map and dendrogram; the heat map represented the standardization coefficient of each component in each sample and the tree graph represented the clustering results.

\section{Quantitative analysis of ganjiang decoction prepared by the five methods}

For the quantitative analysis of ferulic acid, gallic acid, and ellagic acid, the chromatographic conditions were the same as those of the HPLC fingerprint analysis.

For the quantitative analysis of palmatine and berberine hydrochloride, the mobile phases were composed of acetonitrile (A) $-0.1 \%$ phosphoric acid- $0.2 \%$ triethylaminewater (B), with gradient elution (0-15 min, A 20-30\%; 15-35 min, A $30-40 \%$; 35-50 min, A 40-50\%) at a flow rate of $0.5 \mathrm{~mL} / \mathrm{min}$. The column temperature was $25^{\circ} \mathrm{C}$. The detection UV wavelength was $254 \mathrm{~nm}$, and the injection volume was $10 \mu \mathrm{L}$. Sample preparation was the same as that of fingerprint analysis.

Quantitative analysis of zingerone and ligustilide was performed by GC-2010 plus GC System (SHIMADZU Cooperation, Japan) and a Hp-5 column $(30 \mathrm{~m} \times 0.25 \mathrm{~mm} \times 0.25 \mu \mathrm{m})$. Gas column flow rate was $1.0 \mathrm{~mL} / \mathrm{min}$. For the column temperature, the initial temperature was $120{ }^{\circ} \mathrm{C}$, which was maintained for $2 \mathrm{~min}$; subsequently, the temperature was increased to $220{ }^{\circ} \mathrm{C}$ at a rate of $12{ }^{\circ} \mathrm{C} / \mathrm{min}$, which was maintained for $3 \mathrm{~min}$.
Injection port temperature was $250{ }^{\circ} \mathrm{C}$. Detector temperature was $260^{\circ} \mathrm{C}$. Split-flow injection was employed, with a ratio of 20:1 and the injection volume of $1 \mu \mathrm{L}$.

Sample preparation of GC: $1.1 \mathrm{~g}$ of five samples were taken in $11 \mathrm{~mL}$ petroleum ether. Ultrasonic extracted for $30 \mathrm{~min}$. After filtration, petroleum ether was added for extraction again. Two filtrates were combined, concentrated and fixed to $10 \mathrm{ml}$ with petroleum ether. Take the supernatant and pass the $0.22 \mu \mathrm{m}$ microporous membrane to obtain the sample solution.

\section{Animals}

Male C57BL/6 mice (18-22 g) were provided by the Experimental Animals Center of Guangzhou University of Chinese Medicine. The animals were in specific pathogen-free laboratory (temperature $25^{\circ} \mathrm{C}$, relative humidity $60 \%$ ) with alternating light and dark for $12 \mathrm{~h}$ and had free access to tap water and food. This study was approved by the Animal Ethics Committee of Guangzhou University of Chinese Medicine (Approved No. S2018021).

\section{Effect of the different preparation methods of ganjiang decoction on DSS-induced UC in mice}

The mice entered the acute induction period after 7 days of adaptive feeding. Before the experiment, the mice fasted, but could not control the water for $12 \mathrm{~h}$. The mice were provided $3 \%$ DSS solution to drink freely for 7 days to establish the UC model, except those in the control group. After 7 days, the UC mice were divided into the model group (i.g distilled water), SASP group (i.g SASP $0.0038 \mathrm{~g} / 10 \mathrm{~g}$ ), and another five groups for five extracts of the different methods of GD (i.g $0.1001 \mathrm{~g} / 10 \mathrm{~g}$ ) (group 1 (G1), group 2 (G2), group 3 (G3), group 4 (G4), and group 5 (G5)). Control group was given the same amount 
of distilled water. Each group was given gastric gavage once a day for 7 consecutive days.

The gavage drug was prepared according to the "Five preparation methods of ganjiang decoction". Extracts of methods 1-5 was concentrated, the corresponding fine powder was added, mixed into suspension and then gavage. During the modeling and administration period, daily dietary water intake, body weight, and disease active index (DAI) scores (fecal: 0 marks for normal, 1 marks for wet sticky, 2 marks for visible perianal fecal, 3 marks for runny bowel) were recorded. Animals were anesthetized with pentobarbital sodium $24 \mathrm{~h}$ after the last intragastric administration of GD. Blood samples were obtained from the aorta abdominalis and centrifuged at $3000 \mathrm{rpm}$ for $10 \mathrm{~min}$. Subsequently, the serum was aspirated and stored at $-80{ }^{\circ} \mathrm{C}$. The spleen was quickly removed and weighed, and the entire colon was dissociated. The length of the colon was measured, $1 \mathrm{~cm}$ of the diseased colon tissue was cut, and the feces were rinsed; thereafter, the cut tissue was fixed in $4 \%$ paraformaldehyde and stored at $4{ }^{\circ} \mathrm{C}$. The rest of the colon tissue was frozen in liquid nitrogen and stored at $-80{ }^{\circ} \mathrm{C}$ for biochemical analysis.

\section{Histopathological examination and evaluation of colons}

The tissues were fixed in 4\% paraformaldehyde and dehydrated successively by a series of ethanol solutions. The specimens were permeated and paraffin-embedded; thereafter, the specimens were cut $(4 \mu \mathrm{m})$ and stained with hematoxylin-eosin. The stained slices were examined by a pathologist using an Olympus CX31 optical microscope (Olympus Corporation, Tokyo, Japan).

Stained tissues were scored according to the histopathological criteria referenced from Binabaj's study [17]. Histopathological scores were obtained from the sum of the four scores of inflammatory infiltration, crypt loss, mucosal damage and the range of pathological change. The inflammation score was as follows: 0, no inflammatory infiltration; 1 , mild inflammatory infiltration; 2 , moderate inflammatory infiltration; 3 , severe inflammatory infiltration. The crypt loss score was as follows: 0 , normal crypt; 1 , one-third loss; 2 , two-thirds loss; 3 , total crypt loss and intact endothelium; 4, total crypt loss and epithelial loss. Mucosal damage score was as follows: 0 , intact mucosal; 1 , only mucous layer damage; 2 , submucosal injury; 3 , muscle and serous membrane damage. The range score of pathological changes was as follows: 0 , no pathological changed; $1,25-50 \%$ pathological changed; $3,51-75 \%$ pathological changed; $4,76-100 \%$ pathological changed.

\section{Biochemical analysis}

Levels of TNF-a, IL-1 $\beta$, IL-6, IL-10, and IL- $1 \alpha$ in the colon tissue were determined by enzyme-linked immunosorbent assay (ELISA). The Detecting steps assessed using commercially available kits and quantified according to the manufacturers' guidelines (R\&D Systems, LXSAMSM-06).

\section{Statistical analysis}

Statistical analyses were conducted using SPSS 22.0 Statistical Software, and results were treated with GraphPad Prism 5.0. All data were presented as mean \pm standard deviation. Comparison between two groups was examined using the least significant difference (LSD) $t$ test. One-way analysis of variance (ANOVA) followed by the LSD test was used to compare multiple groups. $P<0.05$ was considered statistically significant.

\section{Results}

Fingerprint analysis of the ganjiang decoction extracts by the five methods

The similarity of the fingerprint of the five preparation methods (10 batches) was in the range of $0.990-1.000$ (Table 2).

In the first preparation method, 22 common peaks were marked, of which five common peaks were identified: gallic acid (No. 1 peak), jatrorrhizine (No. 14 peak), berberine hydrochloride (No. 15 peak), palmatine (No. 16 peak) and ellagic acid (No. 23 peak) (Fig. 2a).

In the second preparation method, 22 common peaks were marked, of which seven common peaks were identified: gallic acid (No. 1 peak), jatrorrhizine (No. 14 peak), berberine hydrochloride (No. 15 peak), palmatine (No. 16 peak), ferulic acid (No. 18 peak), zingerone (No. 19 peak), and ellagic acid (No. 23 peak) (Fig. 2b).

In the third preparation method, 22 common peaks were marked, of which seven common peaks were

Table 2 The similarities of ten batches of samples of five extraction methods for ganjiang decoction

\begin{tabular}{llllll}
\hline Sample & \multicolumn{5}{l}{ Similarities } \\
\cline { 2 - 6 } & Method 1 & Method 2 & Method 3 & Method 4 & Method 5 \\
\hline S1 & 0.995 & 0.998 & 0.998 & 1.000 & 0.999 \\
S2 & 0.993 & 0.994 & 1.000 & 1.000 & 0.999 \\
S3 & 0.999 & 0.999 & 1.000 & 0.999 & 1.000 \\
S4 & 0.996 & 0.999 & 1.000 & 1.000 & 1.000 \\
S5 & 0.999 & 1.000 & 1.000 & 1.000 & 1.000 \\
S6 & 0.998 & 0.999 & 0.999 & 1.000 & 1.000 \\
S7 & 0.999 & 1.000 & 1.000 & 0.998 & 1.000 \\
S8 & 0.999 & 0.993 & 0.999 & 1.000 & 1.000 \\
S9 & 0.995 & 0.999 & 0.999 & 1.000 & 1.000 \\
S10 & 0.990 & 0.999 & 1.000 & 0.999 & 1.000
\end{tabular}




\section{a}

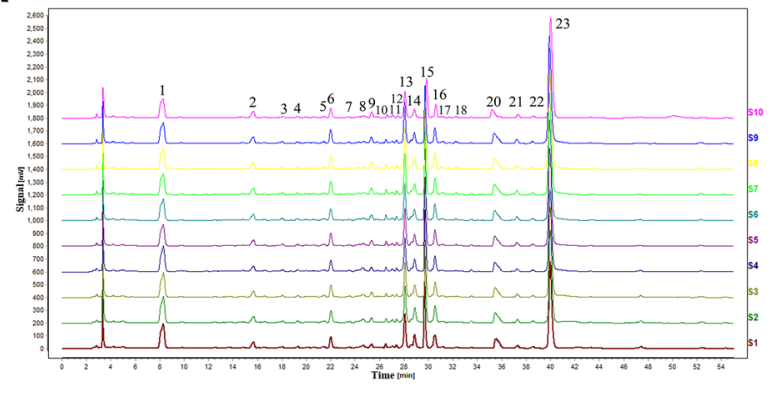

b

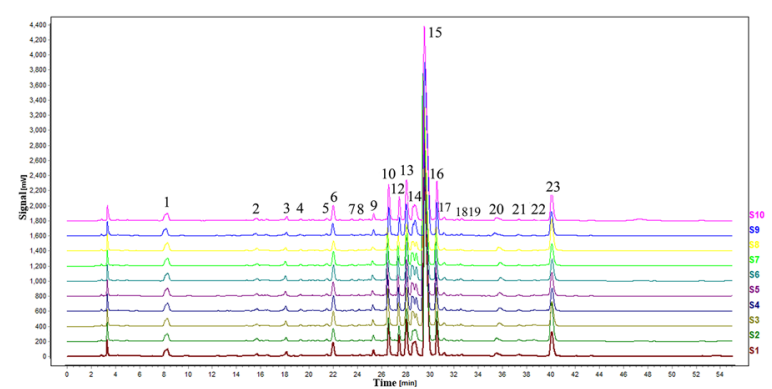

d

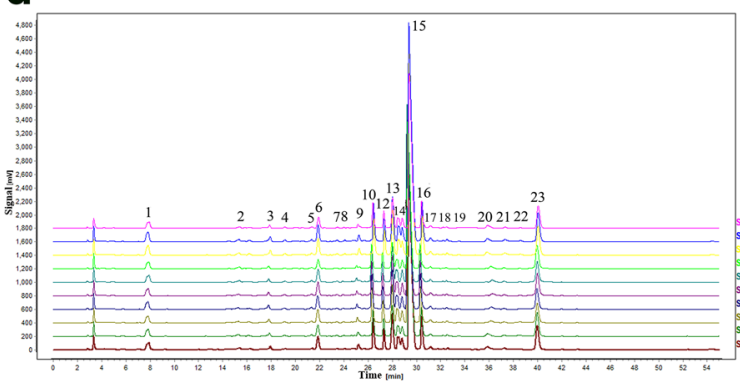

f

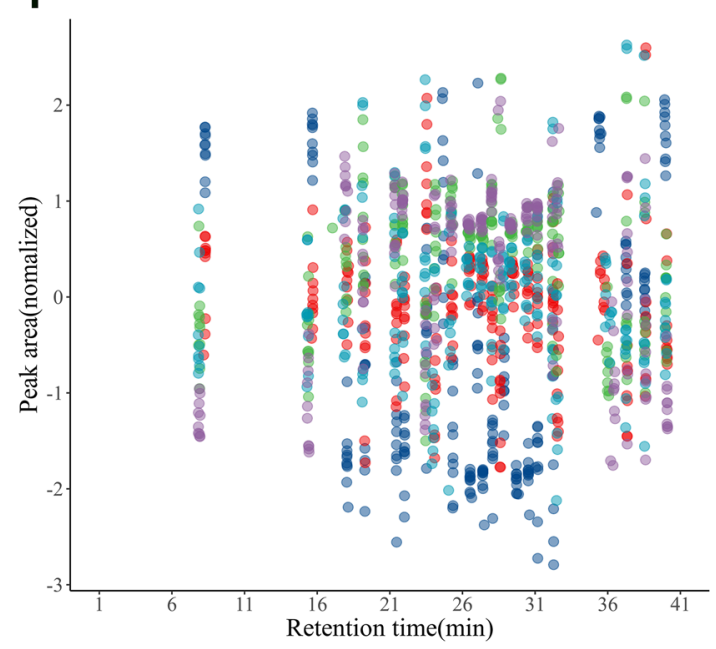

Method $\bullet 1 \bullet 2 \bullet 3 \bullet 4 \bullet 5$
C

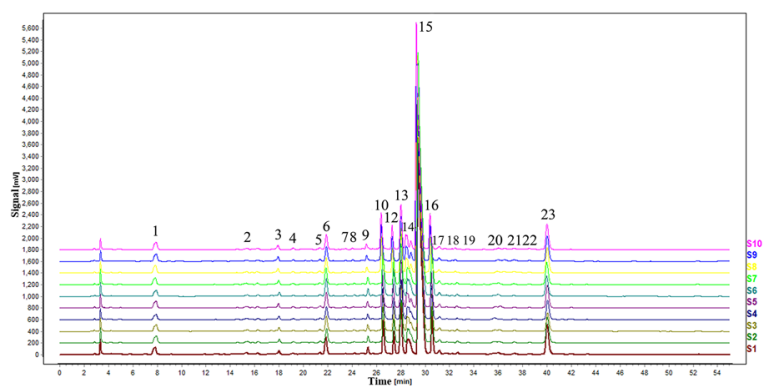

e

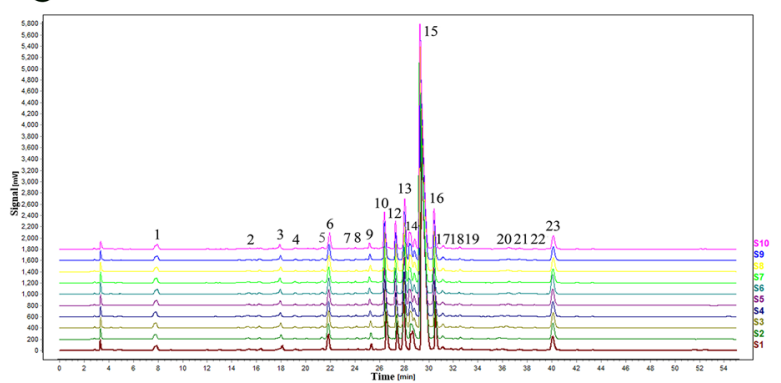

\section{g}

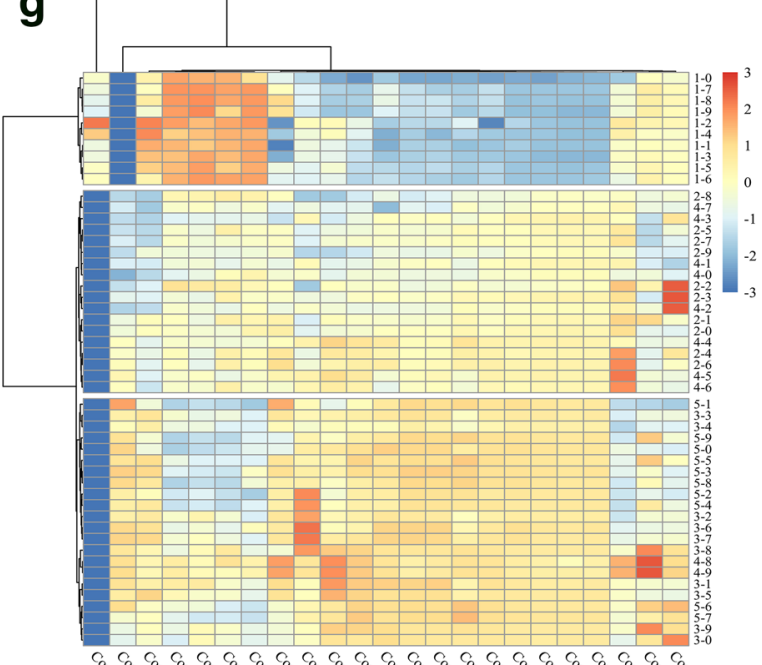


(See figure on previous page.)

Fig. 2 Chromatographic fingerprints and cluster analysis based on the 10 batches of the different extraction of ganjiang decoction. The fingerprint of the extract obtained by the first (a), second (b), third (c), fourth (d), and fifth (e) extraction method of ganjiang decoction. Scatter diagram was plotted according to the retention time and peak area of common peaks (f). Cluster analysis was performed according to the peak area of the

common peak, and the height of peak area was expressed by heat map $(\mathbf{g})$

identified: gallic acid (No. 1 peak), jatrorrhizine (No. 14 peak), berberine hydrochloride (No. 15 peak), palmatine (No. 16 peak), ferulic acid (No. 18 peak), zingerone (No. 19 peak), and ellagic acid (No. 23 peak) (Fig. 2c).

In the fourth preparation method, 22 common peaks were marked, of which seven common peaks were identified: gallic acid (No. 1 peak), jatrorrhizine (No. 14 peak), berberine hydrochloride (No. 15 peak), palmatine (No. 16 peak), ferulic acid (No. 18 peak), zingerone (No. 19 peak), and ellagic acid (No. 23 peak) (Fig. 2d).

In the fifth preparation method, 22 common peaks were marked, of which seven common peaks were identified: gallic acid (No. 1 peak), jatrorrhizine (No. 14 peak), berberine hydrochloride (No. 15 peak), palmatine (No. 16 peak), ferulic acid (No. 18 peak), zingerone (No. 19 peak), and ellagic acid (No. 23 peak) (Fig. 2e).

Scatter diagrams were drawn according to the peak area and retention time of the samples from the 10 batches of the five extraction methods of GD, and different colors represent the different extraction methods (Fig. 2f). Figure 2g showed the results of cluster analysis. The samples were divided into three clusters based on the tree graph: cluster 1 was mainly the sample of method 1; cluster 2, the samples of methods 2 and 4; and cluster 3, the samples of methods 3 and 5 . The analysis indicated that the chemical components extracted by methods 2 and 4 were similar, while those extracted by methods 3 and 5 were similar. Moreover, the heat map also reflected the peak area of each component. The highest contents of compounds 8, 2, 23, 1 , and 20 were found in cluster 1 , which was followed by cluster 2, and the lowest content was noted in cluster 3 . By contrast, compounds $3-6$, compounds $9-10$, and compounds 12-18 had the highest content in cluster 3 , which was followed by cluster 2; the lowest content was found in cluster 1 . In addition, compound 11 only appeared in cluster 1, and compound 19 appeared only in clusters 2 and 3.

\section{Quantitative analysis of ganjiang decoction by HPLC and GC}

Based on the aforementioned results of cluster analysis, compound 1 (gallic acid) and compound 23 (ellagic acid) contents were high in method 1 and low in methods 2-5. The contents of compound 15 (berberine hydrochloride), 16 (palmatine), and 18 (ferulic acid) were the highest in methods 3 and 5 , which was followed by methods 2 and 4; the lowest was noted in method 1. Thus, HPLC was used for quantitative analysis of these components with large content difference. In methods 4 and 5, a small amount of Zingiberis Rhizoma and Angelicae Sinensis Radix fine powder was added; thus, we used GC to quantitatively analyze the volatile components of zingerone and ligustilide.

The reference chromatogram, calibration curves, and linear range of gallic acid, berberine hydrochloride, palmatine, ferulic acid, ellagic acid, zingerone, and ligustilide were shown in Fig. 3 and Table 3.

The quantitative results of gallic acid, berberine hydrochloride, palmatine, ferulic acid, ellagic acid, zingerone, and ligustilide in the GD extracts by the five methods were shown in Table 4. In method 1 , gallic acid and ellagic acid were the highest content, whereas ferulic acid, palmatine, and berberine hydrochloride were the lowest. In methods 3 and 5, as Coptidis Rhizoma and Phellodendri Chinensis Cortex were extracted separately by $50 \%$ alcohol, the alkaloid content, such as palmatine and berberine hydrochloride, was higher. In methods 4 and 5, a small amount of Zingiberis Rhizoma and Angelicae Sinensis Radix powder were added in the extract; thus, the volatile substances, such as zingerone and ligustilide, were higher.

\section{The protective effect of ganjiang decoction in mice with DSS-induced UC}

Mice were treated with $3 \% \mathrm{DSS}$ in water for 7 days to induced an acute UC model. Effect of the GD on DSSinduced colitis was evaluated with the DAI score, colon length, corrected spleen weight, and histopathological analysis of colon tissue.

All the five extractions of GD reduced the DAI scores of mice with UC, especially the first, second and fifth extracts, where the decline in DAI scores was statistically significant (Fig. 4a). Colon appeared extensive hyperaemia oedema and became shorter in mice with UC. Only the fifth extracts significantly increased the colonic length in mice with UC, compared to the model group (Fig. 4b). All the five methods reduced the corrected spleen weight of mice with UC to different degrees; significant corrected spleen weight was noted in the third and fifth methods (Fig. 4c). 

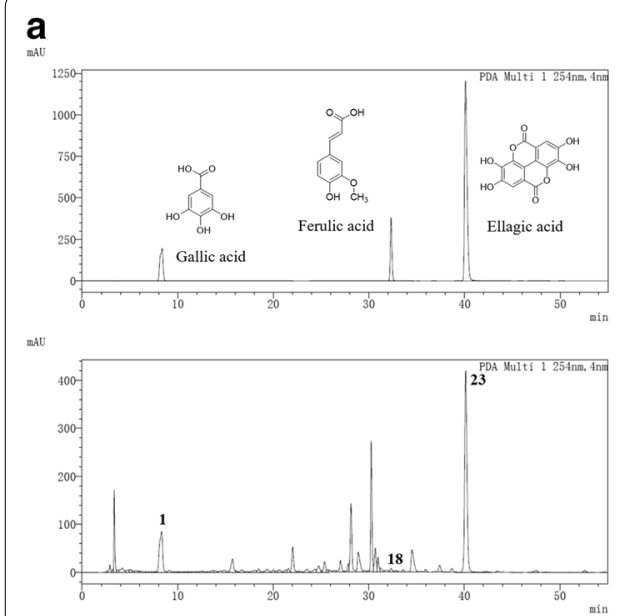

b
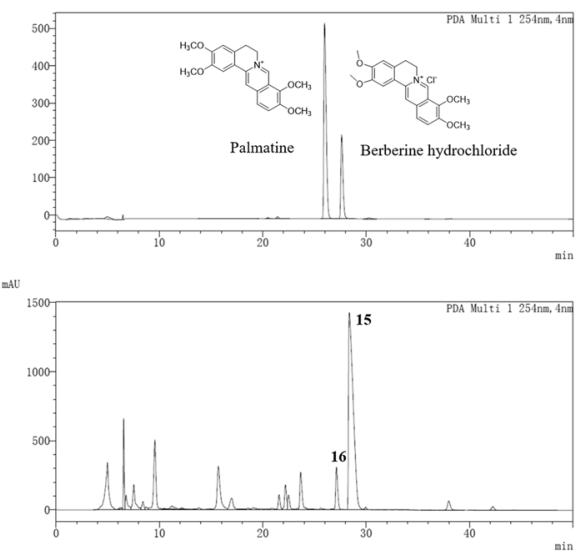

c
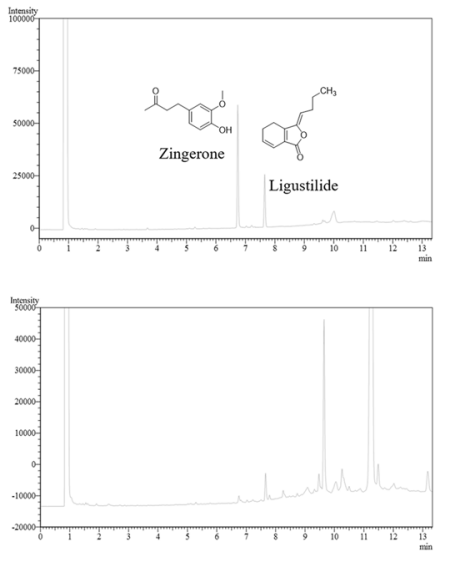

Fig. 3 Reference and sample chromatograms in the quantitative analysis. a Reference and sample chromatograms of gallic acid, ferulic acid, and ellagic acid in the quantitative analysis by HPLC. $\mathbf{b}$ Reference and sample chromatograms of palmatine and berberine hydrochloride in the quantitative analysis by HPLC. c Reference and sample chromatograms of zingerone and ligustilide in the quantitative analysis by GC. HPLC high-performance liquid chromatography, GC gas chromatography

Table 3 Calibration curves

\begin{tabular}{|c|c|c|c|}
\hline Standard substance & Linear relation & $R^{2}$ & Liner range $(\mu \mathrm{g} / \mathrm{mL})$ \\
\hline Ferulic acid & $Y=27206 A-196.23$ & 0.9998 & $1.5625-100$ \\
\hline Gallic acid & $Y=21630 A+39131$ & 0.9999 & $6.25-200$ \\
\hline Ellagic acid & $Y=117421 A-430228$ & 0.9991 & $6.25-200$ \\
\hline Palmatine & $Y=55675 A-70760$ & 0.9994 & $3.125-200$ \\
\hline Berberine Hydrochloride & $Y=13098 A-10110$ & 0.9999 & $3.125-200$ \\
\hline Zingerone & $Y=17424 A-6111.8$ & 0.9984 & $0.3125-20$ \\
\hline Ligustilide & $Y=1437.8 A-1206.1$ & 0.9997 & $1.5625-100$ \\
\hline
\end{tabular}

$\mathrm{Y}$ was the corresponding peak area, and $\mathrm{A}$ was the concentration

Table 4 The content of seven ingredients of different preparations in ganjiang decoction $(\bar{X} \pm S)$

\begin{tabular}{|c|c|c|c|c|c|c|c|}
\hline Method & $\begin{array}{l}\text { Ferulic acid } \\
(\mathrm{mg} / \mathrm{g})\end{array}$ & $\begin{array}{l}\text { Gallic acid } \\
(\mathrm{mg} / \mathrm{g})\end{array}$ & $\begin{array}{l}\text { Ellagic acid } \\
(\mathrm{mg} / \mathrm{g})\end{array}$ & $\begin{array}{l}\text { Palmatine } \\
(\mathrm{mg} / \mathrm{g})\end{array}$ & $\begin{array}{l}\text { Berberine } \\
\text { Hydrochloride } \\
(\mathrm{mg} / \mathrm{g})\end{array}$ & Zingerone $(\mu \mathrm{g} / \mathrm{g})$ & Ligustilide $(\mu \mathrm{g} / \mathrm{g})$ \\
\hline 1 & $0.14 \pm 0.01$ & $4.54 \pm 0.48$ & $3.09 \pm 0.25$ & $1.09 \pm 0.09$ & $21.43 \pm 3.31$ & - & $7.76 \pm 0.26$ \\
\hline 2 & $0.27 \pm 0.03$ & $3.84 \pm 0.07$ & $1.41 \pm 0.03$ & $5.46 \pm 0.89$ & $168.26 \pm 3.63$ & $2.47 \pm 0.07$ & $13.10 \pm 0.30$ \\
\hline 3 & $0.30 \pm 0.01$ & $2.83 \pm 0.03$ & $1.80 \pm 0.19$ & $6.11 \pm 0.45$ & $227.19 \pm 4.79$ & $2.42 \pm 0.01$ & $14.18 \pm 0.36$ \\
\hline 4 & $0.20 \pm 0.02$ & $2.46 \pm 0.07$ & $1.45 \pm 0.12$ & $5.29 \pm 1.10$ & $172.03 \pm 13.67$ & $3.76 \pm 0.07$ & $88.04 \pm 0.95$ \\
\hline 5 & $0.34 \pm 0.05$ & $2.01 \pm 0.17$ & $1.26 \pm 0.18$ & $7.43 \pm 0.39$ & $305.50 \pm 19.58$ & $3.56 \pm 0.04$ & $91.46 \pm 2.73$ \\
\hline
\end{tabular}

$\mathrm{mg} / \mathrm{g}$ and $\mu \mathrm{g} / \mathrm{g}$ refer to the content of components in each gram of dried sample

Pathologic manifestations of UC mice in different groups were shown in Fig. 5. Comparing with the control group, there were pathological changes of the colon in the model group, overall damage to the surface epithelium, disruption of cryptal glands, infiltration of inflammatory cells, the loss of goblet cells was observed in the DSS group. In groups 3, 4, and 5, the intestinal epithelial structure of the mucosal layer was complete, and the epithelial cells were normal and arranged tightly. Crypt inflammation was reduced and infiltration of a few lymphocytes was noted.

\section{Biochemical indexes of mice with DSS-induced UC and the protective effect of ganjiang decoction}

The secretion of inflammatory cytokines in mice with ulcerative colitis is disordered, and pro-inflammatory 

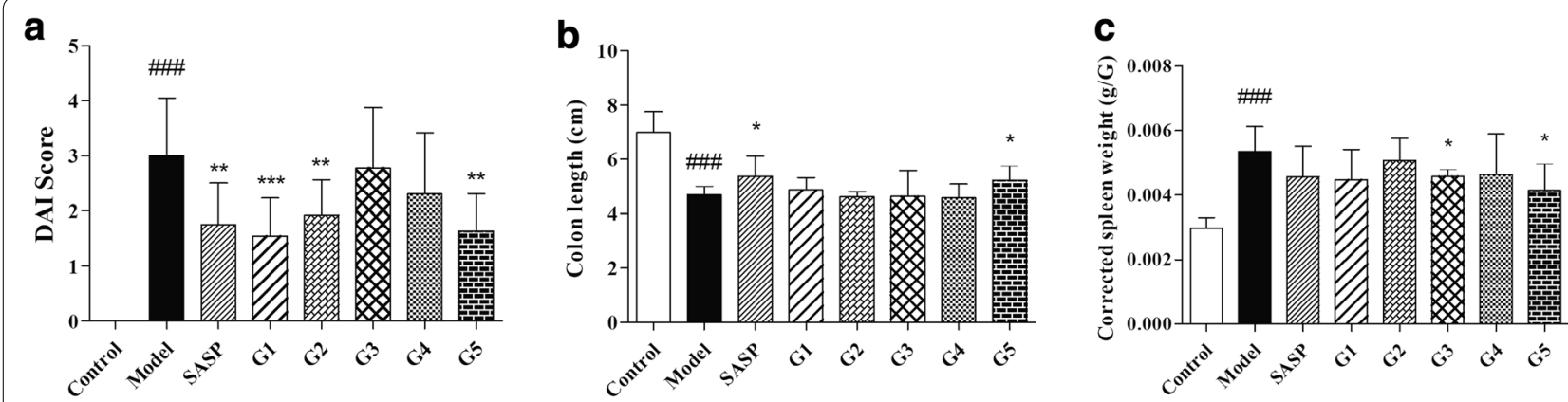

Fig. 4 General efficacy of ganjiang decoction in mice with DSS-induced ulcerative colitis. Effect of different extraction methods of ganjiang decoction on DAI score (a), colonic length (b), and corrected spleen weight (c) in mice with ulcerative colitis. Data were presented as mean $\pm S D$

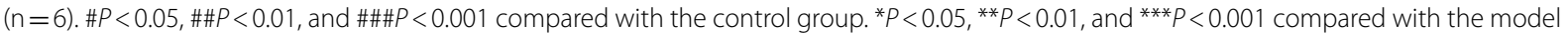
group. DSS dextran sulphate sodium salt, DAl disease activity index, SD standard deviation

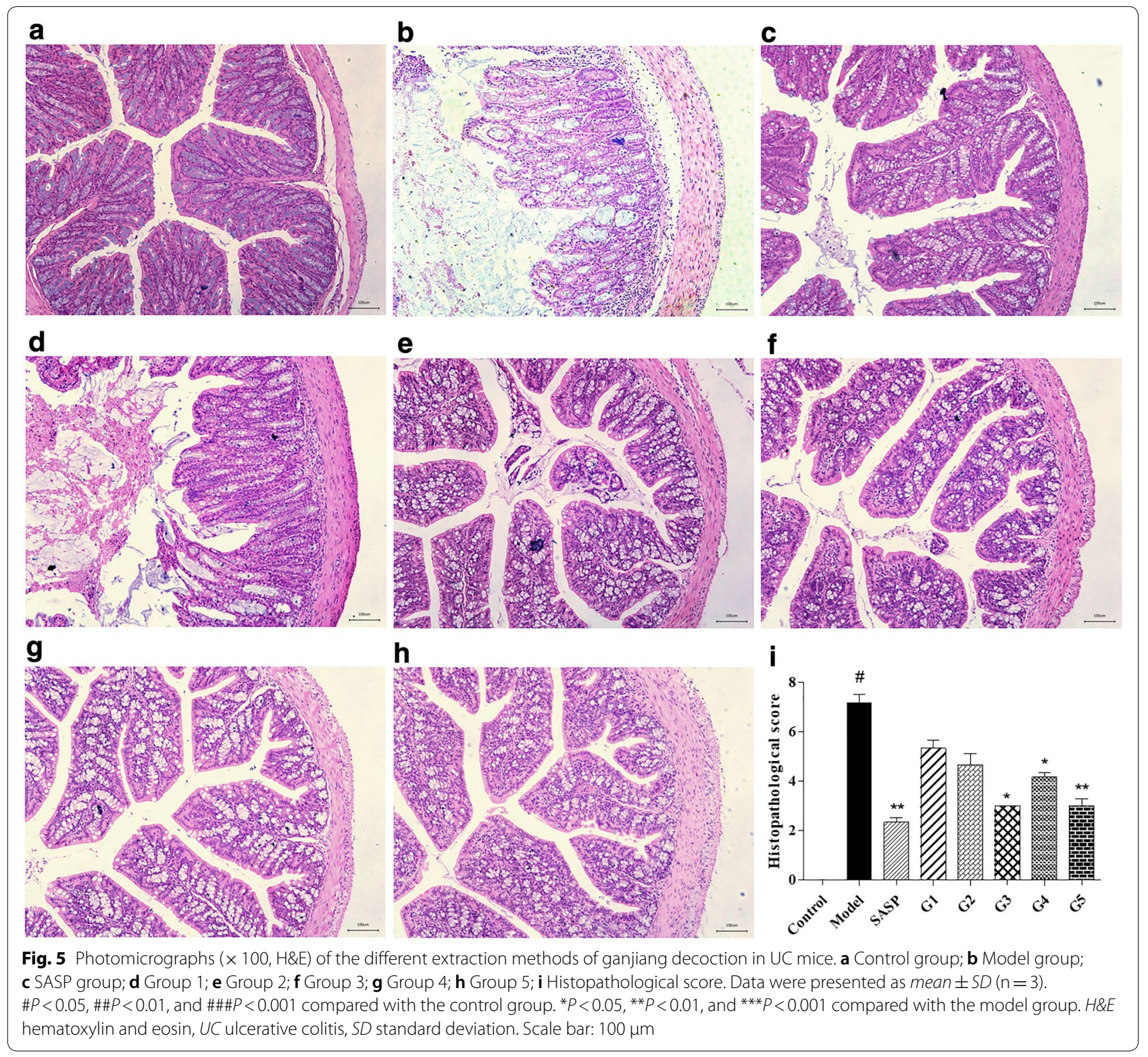



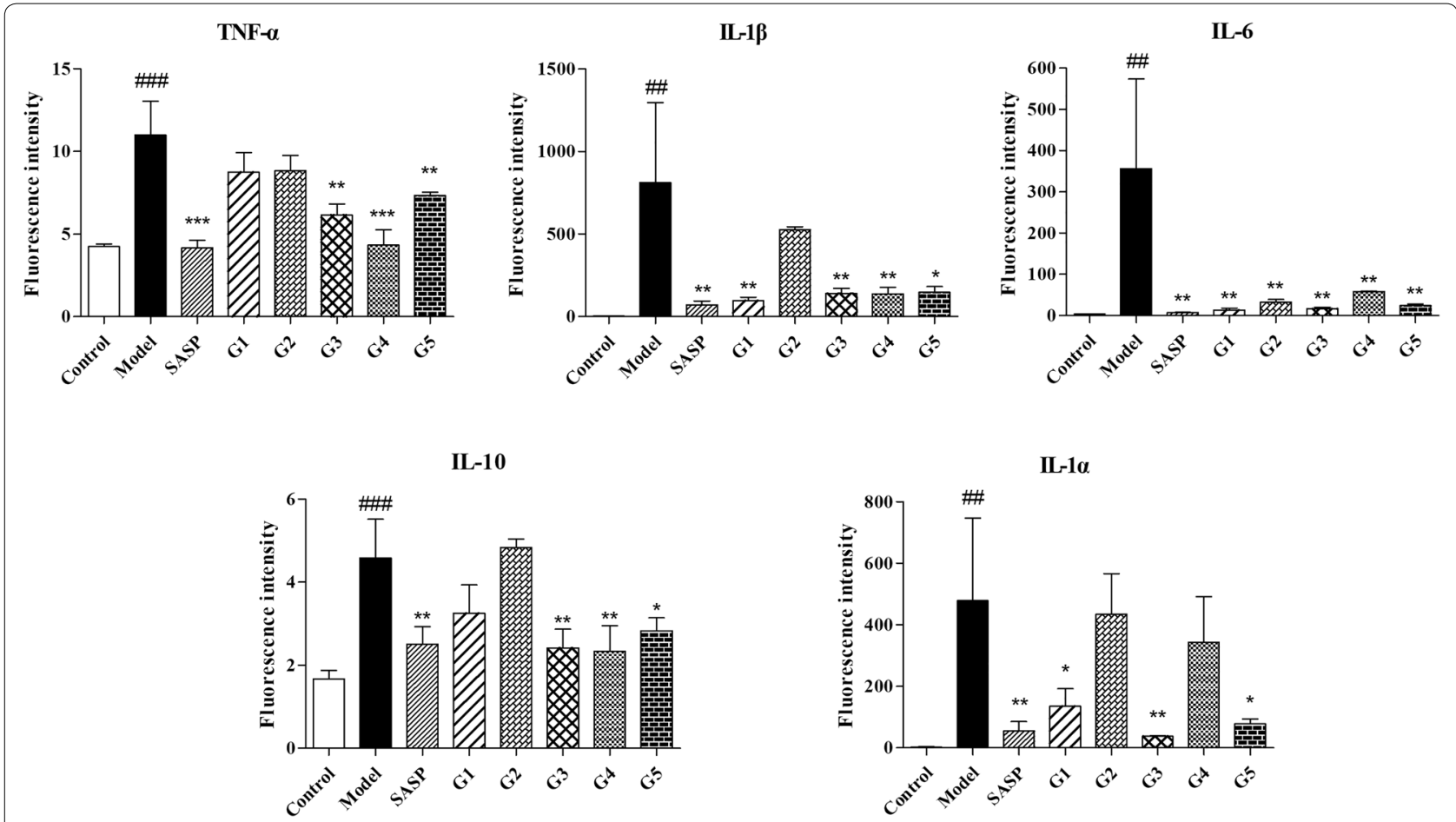

Fig. 6 Effects of the five preparation methods of ganjiang decoction on colonic inflammatory factors in UC mice. Data were presented as mean $\pm S D(n=6)$. $\# P<0.05, \# \# P<0.01$, \#\#\#P<0.001 compared with the control group. ${ }^{*} P<0.05,{ }^{* *} P<0.01,{ }^{* * *} P<0.001$ compared with the model group. UC ulcerative colitis, $S D$, standard deviation

factors increases dramatically. Inflammatory cytokines of mice with DSS-induced UC and the protective effect of GD were shown in Fig. 6.

All the five extractions of GD reduced the levels of TNF- $\alpha$ of mice with UC, especially the third, fourth and fifth extracts, where TNF- $\alpha$ level was reduced significantly; the other methods showed a reduction tendency. The extract of method $1,3,4$, and 5 could notably reduce the IL- $1 \beta$ level in the colon tissues of the UC mice. All the five extraction methods could significantly reduce the IL-6 level in the colon tissues of the mice. IL-10 levels were significantly decreased in the third, fourth, and fifth groups. The first, third, and fifth extraction methods reduced the IL- $1 \alpha$ level in the colon tissues of the mice significantly.

\section{Discussion}

GD was first recorded in the 'Thousand-Golden-Prescriptions (Beiji Qianjin Yao Fang), which was mainly used for the treatment of small intestine distension and pain, hematochezia or loose stools, and intestinal peristalsis, which are clinical manifestations similar to those of UC. Since the onset of UC is mostly in the colon and rectum, which cannot be reached by traditional decoction, we considered to develop GD as a colonic targeted preparation to improve its efficacy. The traditional Chinese medicine decoction contains a variety of ingredients, so it is necessary to make a research on the formulation and selection of ingredients before the preparation development. In this study, pharmacological efficacy was used as the evaluation index to explore and improve the extraction method of GD. Our results showed that the five extraction methods of GD improved the DSS-induced UC in mice to different degrees, and the difference in efficacy was closely related to the extraction methods.

\section{The increase of alkaloid and volatile oil affected the effect of GD on UV mice}

The first extraction method of GD is the most common extraction method of traditional Chinese medicine prescriptions. The cluster and quantitative analyses showed that the content of the chemical components obtained by the first extraction method was significantly different from that obtained by the other four extraction methods. This method did not take into account the relationship between the phytochemical constituents of the Chinese medicinal herbs and the dissolution of active ingredients by an extraction solvent. 
Coptidis Rhizoma and Phellodendri Chinensis Cortex contain alkaloids $[18,19]$, while Granati Pericarpium and Sanguisorbae Radix contain tannins [7, 20]. Alkaloids can react with tannins to form the insoluble precipitate, thus reducing the dissolution efficiency of alkaloids. Hence, in the second, third, fourth, and fifth methods, Coptidis Rhizoma and Phellodendri Chinensis Cortex were extracted by water and $50 \%$ ethanol separately, and the alkaloid content was significantly increased. Literature had shown that the extraction efficiency of alkaloids was the highest when the concentration of ethanol was $50 \%-60 \%$ [18]. As 50\% ethanol was used as the extraction solvent of Coptidis Rhizoma and Phellodendri Chinensis Cortex, which further improved the extraction efficiency of alkaloids; and the separate extraction of Sanguisorbae Radix and Granati Pericarpium reduced the influence of tannins on alkaloids. Therefore, the palmatine and berberine hydrochloride contents were highest in the third and fifth extraction methods. Studies showed that berberine hydrochloride had beneficial effects on UC, such as suppression of IL-1, IL-1 $\beta$, IL-6, IL-12, and TNF- $\alpha$ expression $[4,6]$. Palmatine also showed an anti-inflammatory action $[2,5]$. The high alkaloid content might be the reason why the third and fifth extracts were superior to other extracts in reducing inflammatory factors.

The third and fifth extracts showed the best protective effect against ulcerative colitis based on pathological sections and biochemical indicators. Nevertheless, the fifth method was more advantageous not only considering the DAI score, the length of the colon and the relative weight of the spleen but also because of the greater alkaloids and volatile components in the extract.

In the later stage, we plan to make GD into intestinal soluble pellets. In the optimization of preparation method, the volatile oil of Zingiberis Rhizoma and Angelicae Sinensis Radix was not extracted by reflux method, but some fine powder was added, which could not only improve the content of volatile components, but also reduce the use of excipients in the preparation of pellets. Therefore, the fourth and fifth extraction methods were improved on the basis of the second and third methods. This improvement was to pulverize part of Zingiberis Rhizoma and Angelicae Sinensis Radix into fine powder and directly add into the extraction solution, retaining volatile components such as zingerone and ligustilide. Moreover, the quantitative analysis revealed that zingerone and ligustilide were detected in the second, third, fourth, and fifth extraction methods, and the highest contents were found in the fourth and fifth extraction methods after the addition of Zingiberis Rhizoma and Angelicae Sinensis Radix fine powder.
The fifth extraction method had the highest ferulic acid and ligustilide contents and higher zingerone. Ferulic acid showed a good antioxidant effect [21, 22], and ligustilide played a positive role in lipopolysaccharideinduced inflammation in RAW 264.7 macrophages and attenuated vascular inflammation and in activating the defense system of endothelial cells [23, 24]. Moreover, ligustilide showed a good analgesic activity, which inhibited the activation of JNK/c-Jun in spinal cord caused by complete freudian adjuvant (CFA) [25], reduced the inflammatory pain of spinal astrocytes after CFA peripheral injection [26], suppressed microglia cells to mediate inflammatory pain [27]. Other studies reported that ferulic acid and ligustilide could achieve the therapeutic effect on cold-induced vasospasm through the combined action of TRPM8 and TPRA1 [28], which might also be a mechanism for the analgesic effect of the fifth extract. Thus, the increase in these components could explain the better integrated therapeutic effect of the fifth extract on mice with UC.

\section{Chinese herbal compound prescription has become a new trend in the treatment of UC}

SASP has a good therapeutic effect on UC, but there are many reports of adverse reactions, the most common adverse reactions are anal stimulation and abdominal pain [29]. Chinese herbal compound prescription has become a new trend in the treatment of UC because of its advantages of multi-component and multi-target with little side effect. Gegenqinlian decoction could maintain colonic mucosal homeostasis in acute/chronic ulcerative colitis via Notch signal pathway by bidirectional regulation [30]. Sanhuang shu'ai decoction alleviated DSS-induced ulcerative colitis by regulating intestinal microbial flora, inflammatory mediators and cytokines [31]. Studies had shown that many traditional Chinese medicine compounds and formulations could act on NF-k B p65 directly or indirectly to improve the symptoms of UC [32]. For example, huangqin decoction inhibited the anti-inflammatory effect of RASPI3K-AKT-HIF- $1 \alpha$ and NF-kB pathway on the UC mice by regulating the intestinal microflora [33]. Huanglian jiedu decoction could alleviate the damage of intestinal mucosal and protect the UC mice by downregulating JAK2 and STAT3 expression via JAK2/STAT3 pathway [34]. In this paper, the extraction method of GD had been improved. The extract of the fifth method contained a variety of active ingredients, which could play a role in multiple phenotypes of UC such as inflammation and abdominal pain, showing the advantages of multi-target of traditional Chinese medicine compound prescription. 


\section{Conclusions}

This study showed an improved extraction process of GD based on the fingerprint cluster analysis and content determination and on the therapeutic effect on UC in mice. The results showed that the difference of the five extracts of GD in the efficacy of DSS-induced UC in mice was closely related to the extraction method. These results provided a basis for the enteric preparation of GD and a new idea for traditional Chinese medicine compound preparation.

\begin{abstract}
Abbreviations
UC: Ulcerative colitis; GD: Ganjiang decoction; HPLC: High-performance liquid chromatography; DSS: Dextran sulphate sodium salt; GC: Gas chromatography; SASP: Salicylazosulfapyridine; i.g: Intragastric administration; DAl: Disease active index; H\&E: Hematoxylin-eosin; ELISA: Enzyme-linked immunosorbent assay; LSD: Least significant difference; ANOVA: One-way analysis of variance; TNF-a: Tumor necrosis factor $\alpha$; IL-1 $\beta$ : Interleukin 1 $\beta$; IL-6: Interleukin 6; IL-10: Interleukin 10; IL-1a: Interleukin 1a.
\end{abstract}

\section{Acknowledgements}

Not applicable.

\section{Authors' contributions}

YY and TML were responsible for the concept and design of this study. YYW, $\mathrm{NJ}$ and TL performed the experiments. YYW, NJ and LKL analyzed data. YYW embellished the figures and drafted the manuscript. CL and WX offered help and participated in experimental discussions. All authors read and approved the final manuscript.

\section{Funding}

This work was supported by the Guangzhou University of Chinese Medicine 'High-level university construction project' (Grant No: A1-AFD018191A33).

\section{Availability of data and materials}

Not applicable.

\section{Ethics approval and consent to participate}

This study was approved by the Animal Ethics Committee of Guangzhou University of Chinese Medicine (Approved No. S2018021).

\section{Consent for publication}

All authors critically reviewed the content of the manuscript. The consent for publication was obtained from all authors.

\section{Competing interests}

No potential conflict of interest was reported by the authors.

\section{Author details}

${ }^{1}$ School of Pharmaceutical Sciences, Guangzhou University of Chinese Medicine, Guangzhou, Guangdong 510006, China. ${ }^{2}$ The Second Affiliated Hospital of Guangzhou, University of Chinese Medicine, Guangzhou, Guangdong 510006, China.

Received: 20 April 2020 Accepted: 20 July 2020

Published online: 05 August 2020

\section{References}

1. Chrubasik S, Pittler MH, Roufogalis BD. Zingiberis rhizoma: a comprehensive review on the ginger effect and efficacy profiles. Phytomedicine. 2005;12(9):684-701.

2. Mai C, Wu M, Wang C, Su Z, Cheng Y, Zhang X. Palmatine attenuated dextran sulfate sodium (DSS)-induced colitis via promoting mitophagy-mediated NLRP3 inflammasome inactivation. Mol Immunol. 2019;105:76-85.
3. Cui HT, Cai YZ, Wang L, Jia BT, Li JC, Zhao SW, et al. Berberine regulates Treg/Th17 balance to treat ulcerative colitis through modulating the gut microbiota in the colon. Front Pharmacol. 2018;9:17.

4. Zhu L, Gu P, Shen H. Protective effects of berberine hydrochloride on DSS induced ulcerative colitis in rats. Int Immunopharmacol. 2019;68:242-51.

5. Zhang X, Yuan Z, Qu C, Yu X, Huang T, Chen P, et al. Palmatine ameliorated murine colitis by suppressing tryptophan metabolism and regulating gut microbiota. Pharmacol Res. 2018;137:34-46.

6. Liao Z, Xie Y, Zhou B, Zou B, Xiao D, Liu W, et al. Berberine ameliorates colonic damage accompanied with the modulation of dysfunctional bacteria and functions in ulcerative colitis rats. Appl Microbiol Biotechnol. 2020;104(4):1737-49.

7. Wang BS, Leu KL, Huang GJ, Yeh CF, Tai HM, Ho WY, et al. Protective effects of an aqueous Pericarpium Granati extract against inflammatory damage in mice. J Funct Food. 2014;9:183-91.

8. Yang JH, Yoo JM, Cho WK, and Ma JY. Ethanol Extract of Sanguisorbae Radix Inhibits Mast Cell Degranulation and Suppresses 2,4-Dinitrochlorobenzene-Induced Atopic Dermatitis-Like Skin Lesions. Mediat. Inflamm. 2016:8.

9. Burisch J. Crohn's disease and ulcerative colitis. Dan Med J. 2014;61(1):32.

10. Molodecky NA, Soon IS, Rabi DM, Ghali WA, Ferris M, Chernoff G, et al. Increasing incidence and prevalence of the inflammatory bowel diseases with time, based on systematic review. Gastroenterology. 2012;142(1):46-54.

11. Ungaro R, Mehandru S, Allen PB, Peyrin-Biroulet L, Colombel J-F. Ulcerative colitis. Lancet. 2017;389(10080):1756-70.

12. Smillie C, Biton M, Ordovas-Montanes J, Sullivan K, Burgin G, Graham D, et al. Intra- and inter-cellular rewiring of the human colon during ulcerative colitis. Cell. 2019;178(3):714-730.e722.

13. Kayal M and Shah S. Ulcerative Colitis Current and Emerging Treatment Strategies Journal of Clinical Medicine. 2019; 9(94).

14. Sun $H$, Zhang AH, Yang L, Li MX, Fang H, Xie J, et al. High-throughput chinmedomics strategy for discovering the quality-markers and potential targets for Yinchenhao decoction. Phytomedicine. 2019;54:328-38.

15. Tao WY, Xu X, Wang X, Li BH, Wang YH, Li Y, et al. Network pharmacologybased prediction of the active ingredients and potential targets of Chinese herbal Radix Curcumae formula for application to cardiovascular disease. J Ethnopharmacol. 2013;145(1):1-10.

16. Xu J, Lian FM, Zhao LH, Zhao YF, Chen XY, Zhang $X$, et al. Structural modulation of gut microbiota during alleviation of type 2 diabetes with a Chinese herbal formula. ISME J. 2015;9(3):552-62.

17. $M M B, F A, A$ A, F R, A S, MR P, et al. EW-7197 prevents ulcerative colitisassociated fibrosis and inflammation. Journal of cellular physiology. 2019; 234(7):11654-11661.

18. Meng FC, Wu ZF, Yin ZQ, Lin LG, Wang RB, Zhang QW. Coptidis rhizoma and its main bioactive components: recent advances in chemical investigation, quality evaluation and pharmacological activity. Chin Med. 2018;13:18.

19. Sun Y, Lenon GB, and Yang AWH. Phellodendri Cortex: A Phytochemical, Pharmacological, and Pharmacokinetic Review. Evid.-based Complement Altern. Med. 2019:45.

20. Seo CS, Jeong SJ, Yoo SR, Lee NR, Shin HK. Quantitative analysis and in vitro anti-inflammatory effects of gallic acid, ellagic acid, and quercetin from radix sanguisorbae. Pharmacogn Mag. 2016;12(46):104-8.

21. Bumrungpert A, Lilitchan S, Tuntipopipat S, Tirawanchai N, Komindr S. Ferulic acid supplementation improves lipid profiles, oxidative stress, and inflammatory status in hyperlipidemic subjects: a randomized, doubleblind, placebo-controlled clinical trial. Nutrients. 2018;10(6):8.

22. Zdunska K, Dana A, Kolodziejczak A, Rotsztejn H. Antioxidant properties of ferulic acid and its possible application. Skin Pharmacol Physiol. 2018;31(6):332-6.

23. Choi ES, Yoon JJ, Han BH, Jeong DH, Lee YJ, Kang DG, et al. Ligustilide attenuates vascular inflammation and activates $\mathrm{Nrf} 2 / \mathrm{HO}-1$ induction and NO synthesis in HUVECs. Phytomedicine. 2018;38:12-23.

24. Su YW, Chiou WF, Chao SH, Lee MH, Chen CC, Tsai YC. Ligustilide prevents LPS-induced iNOS expression in RAW macrophages by preventing ROS production and down-regulating the MAPK, NF-kappa B and AP-1 signaling pathways. Int Immunopharmacol. 2011;11(9):1166-72.

25. Wang $Y, X u H$, Tao M, Xu L, Fu X. Ligustilide relieves complete freund's adjuvant-induced mechanical hyperalgesia through inhibiting the 
activation of spinal c-Jun N-terminal Kinase/c-Jun pathway in rats. Pharmacogn Mag. 2017;13(52):634-8.

26. Qian B, Li F, Zhao L, Dong Y, Gao Y, Zhang Z. Ligustilide ameliorates inflammatory pain and inhibits TLR4 upregulation in spinal astrocytes following complete freund's adjuvant peripheral injection. Cell Mol Neurobiol. 2016;36(1):143-9.

27. Zhu M, Zhao L, Wang X, Gao Y, Zhang Z. Ligustilide inhibits microgliamediated proinflammatory cytokines production and inflammatory pain. Brain Res Bull. 2014:109:54-60

28. Pan Y, Zhao G, Cai Z, Chen F, Xu D, Huang S, et al. Synergistic Effect of Ferulic Acid and Z-Ligustilide, Major Components of A. sinensis, on Regulating Cold-Sensing Protein TRPM8 and TPRA1 In Vitro. Evidence-based complementary and alternative medicine: eCAM. 2016; 2016:3160247.

29. Marshall J, Thabane M, Steinhart A, Newman J, Anand A, and Irvine E. Rectal 5-aminosalicylic acid for maintenance of remission in ulcerative colitis. The Cochrane database of systematic reviews. 2012; 11:CD004118.

30. Zhao Y, Luan H, Gao H, Wu X, Zhang Y, Li R. Gegen Qinlian decoction maintains colonic mucosal homeostasis in acute/chronic ulcerative colitis via bidirectionally modulating dysregulated Notch signaling. Phytomedicine Int J Phytother Phytopharmacol. 2020;68:153182.

31. Wu Z, Zhao Z, Deng J, Huang J, Wang Y, and Wang Z. Sanhuang Shu'ai decoction alleviates DSS-induced ulcerative colitis via regulation of gut microbiota, inflammatory mediators and cytokines. Biomedicine \& pharmacotherapy $=$ Biomedecine \& pharmacotherapie. 2020; 125:109934
32. Lu PD, Zhao YH. Targeting NF-kappa B pathway for treating ulcerative colitis: comprehensive regulatory characteristics of Chinese medicines. Chin Med. 2020;15(1):25.

33. Li M, Luo H, Wu X, Liu Y, Gan Y, Xu N, et al. Anti-inflammatory effects of huangqin decoction on dextran sulfate sodium-induced ulcerative colitis in mice through regulation of the gut microbiota and suppression of the Ras-PI3K-Akt-HIF-1a and NF-KB pathways. Front Pharmacol. 2019:10:1552.

34. Lu Z, Xiong WN, Xiao SM, Lin YL, Yu K, Yue GH, et al. Huanglian Jiedu Decoction ameliorates DSS-induced colitis in mice via the JAK2/STAT3 signalling pathway. Chin Med. 2020;15(1):12.

\section{Publisher's Note}

Springer Nature remains neutral with regard to jurisdictional claims in published maps and institutional affiliations.
Ready to submit your research? Choose BMC and benefit from:

- fast, convenient online submission

- thorough peer review by experienced researchers in your field

- rapid publication on acceptance

- support for research data, including large and complex data types

- gold Open Access which fosters wider collaboration and increased citations

- maximum visibility for your research: over $100 \mathrm{M}$ website views per year

At BMC, research is always in progress.

Learn more biomedcentral.com/submissions 